\title{
0335 DIFFERENT OUTCOMES FOR SIMILAR MECHANISMS OF INJURY: A COMPARISON BETWEEN TWO GROUPS OF INTENTIONAL INJURIES
}

M Avitzour*, R Cohen, K Peleg Correspondence: National Center for Trauma and Emergency Medicine Research, The Gertner Institute, Tel Hashomer 52621, Israel

\subsection{6/ip.2010.029215.335}

Firearms, cuts and falls from heights are the major causes of injury among hospitalised of aggressive attempted suicide (AAS) and victims of violence (VV).

Aim To compare the outcomes of similar mechanisms of injury for patients of AAS and VV.

Method A retrospective study using data from the Israel National Trauma Registry including 10 hospitals during the years 2003-2007. ICD9 definitions of AAS and VV were utilised.

Results During the study period, 825 AAS patients and 7912 VV patients were hospitalised. The most common mechanism of injury for VV was stab wounds (40\%) and non-firearm fighting (28\%). The outcome was different, among AAS, $45 \%$ jumped from height and $32 \%$ from stab wounds. For both groups, approximately $10 \%$ were injured from firearms. AAS patients were more severe, $39 \%$ had an ISS of $16+, 45 \%$ were hospitalised for $7+$ days and $31 \%$ were admitted to the ICU, compared to $16 \%, 16 \%$ and $9 \%$ respectively among VV. In general, VV were younger than AAS. In-hospital mortality was 8.1 times greater among AAS victims, and similarly, rehabilitation referrals were 10 times greater among AAS patients.

Conclusions and Recommendations (1) Hospitalised AAS patients are severely injured, and require increased use of hospital resources. Further research should check if this group receives the necessary support during hospitalisation and follow up treatment after discharge. (2) Methods for reducing use of aggressive mechanisms of violence should begin with the local municipality taking responsibility and working together with other forces. 